\title{
Associations between Cerebral Embolism and Carotid Intraplaque Hemorrhage during Protected Carotid Artery Stenting
}

\author{
(D) G.H. Chung, (D).Y. Jeong, (D)H.S. Kwak, and DS.B. Hwang
}

\begin{abstract}
BACKGROUND AND PURPOSE: Carotid artery stent placement in patients with intraplaque hemorrhage remains controversial because of the incidence of cerebral embolism after the procedure. The purpose of this study is to determine if intraplaque hemorrhage is a significant risk factor for cerebral embolism during carotid artery stent placement.
\end{abstract}

MATERIALS AND METHODS: This prospective study assessed 94 consecutive patients with severe carotid stenosis. These patients underwent preprocedural carotid MR imaging and postprocedural DWI after carotid artery stent placement. Intraplaque hemorrhage was defined as the presence of high signal intensity within the carotid plaque that was $>200 \%$ of the signal from the adjacent muscle on MPRAGE. We then analyzed the incidence of postprocedural ipsilateral ischemic events on DWI and primary outcomes within 30 days of carotid artery stent placement.

RESULTS: Forty-three patients (45.7\%) had intraplaque hemorrhage on an MPRAGE image. There was no significant difference in the incidence of postprocedural ipsilateral ischemic events and primary outcomes between the intraplaque hemorrhage and non-intraplaque hemorrhage group. However, postprocedural ipsilateral ischemic events were more frequently observed in the symptomatic group (17/41 [41.5\%]) than in the asymptomatic group (8/53 [15.1\%]; $P=.005)$.

CONCLUSIONS: Intraplaque hemorrhage was not a significant risk factor for cerebral embolism during carotid artery stent placement in patients with severe carotid stenosis. Symptomatic patients should receive more careful treatment during carotid artery stent placement because of the higher risk of postprocedural ipsilateral ischemic events.

ABBREVIATIONS: CAS = carotid artery stent placement; IPH = intraplaque hemorrhage

$\mathbf{E}^{\mathrm{x}}$ tracranial carotid artery stenosis is considered a causative fac- tor in $20 \%-30 \%$ of all strokes. ${ }^{1-3}$ Large randomized clinical trials showed that carotid endarterectomy is superior to carotid artery stent placement (CAS) for the management of carotid artery stenosis. ${ }^{4-6}$ Other randomized clinical trials showed that CAS and carotid endarterectomy offer similar efficacy. ${ }^{7}$ Although indications for CAS remain controversial, CAS has emerged as a less-invasive treatment that requires shorter hospital times than carotid endarterectomy.

Some studies found a relationship between the baseline pres-

Received May 11, 2015; accepted after revision July 29.

From the Radiology and Research Institute, Clinical Medicine of Chonbuk National University-Biomedical Research Institute of Chonbuk National University Hospital, Jeollabuk-do, Republic of Korea.

Please address correspondence to Hyo Sung Kwak, MD, Radiology and Research Institute of Clinical Medicine of Chonbuk National University-Biomedical Research Institute of Chonbuk National University Hospital, 567 Baekje-daero, deokjin-gu, Jeonju-si, Jeollabuk-do, 561-756, Republic of Korea; e-mail: kwak8140@jbnu.ac.kr; @kwakhs8140

http://dx.doi.org/10.3174/ajnr.A4576 ence of carotid intraplaque hemorrhage (IPH) and the development of ischemic stroke in previously asymptomatic and symptomatic patients. ${ }^{8-10} \mathrm{IPH}$ is associated with plaque progression and, consequently, induces luminal narrowing. Thus, IPH may serve as a measure of risk for the development of future ischemic stroke. The risk of cerebral embolism after CAS in patients with IPH is controversial. Yoshimura et $\mathrm{al}^{11}$ reported that a high-intensity signal on TOF MRA indicates that carotid plaques are at high risk for cerebral embolism during stent placement. However, Yoon et $\mathrm{al}^{12}$ reported that protected CAS seems to be safe in patients with severe carotid stenosis and IPH. This study did not perform DWI to evaluate ipsilateral ischemic lesions. In addition, these studies used TOF imaging to detect IPH. Alternative techniques proposed for more accurate detection of IPH include heavily T1-weighted techniques, such as the MPRAGE sequence. Ota et $\mathrm{al}^{13}$ reported that the MPRAGE sequence demonstrated higher diagnostic capability in detecting IPH when compared with conventional T1-weighted sequences or TOF sequences.

We prospectively designed the study with the following in- 
clusion criteria: 1) preoperative multicontrast carotid plaque MR; 2) protected CAS; 3) postprocedural imaging, including DWI and noncontrast CT within 24 hours; and 4) clinical outcomes after 30 days. The aim of this study was to determine whether IPH is a significant risk factor for cerebral embolism during CAS.

\section{MATERIALS AND METHODS Study Population}

This prospective study was conducted with institutional review board approval. We included 94 consecutive patients with carotid artery stenosis between April 2013 and January 2015. All the patients had symptomatic carotid artery stenosis of $>50 \%$ (NASCET criteria) or asymptomatic carotid artery stenosis of $>70 \%$ (NASCET criteria). Stenosis was initially detected in symptomatic patients by a stroke MR protocol that included contrast-enhanced MR angiography. Stenosis was diagnosed in asymptomatic patients by carotid sonography or/and a routine brain MR imaging that included contrast-enhanced MR angiography to evaluate neurologic symptoms. Multicontrast carotid plaque MR imaging was performed within 3 days before CAS, and new postoperative ischemic lesions were assessed by DWI within 2 days after CAS.

\section{Stroke MR and Multicontrast Carotid Plaque Imaging}

All MR imaging was acquired on a 3T scanner (Achieva; Philips Medical Systems, Best, the Netherlands) with a 16-channel head coil. Stroke MR imaging was performed immediately after CT scanning with the following techniques: DWI, 3D TOF MRA of the intracranial arteries, susceptibility-weighted imaging, perfusion-weighted imaging, and contrast-enhanced MRA for evaluation of carotid arteries. The total scan time was approximately 20-30 minutes. Our protocol for multicontrast carotid plaque imaging included 5 different axial scans: TOF, T1-weighted, T2weighted, postcontrast T1-weighted, and MPRAGE sequences. All the sequences were centered at the bifurcation of the index artery with the carotid plaque. T1-weighted, T2-weighted, and postcontrast $\mathrm{T} 1$-weighted sequences were obtained with a 2.0-mm section thickness and no intersection spacing. TOF and MPRAGE sequences had a 1.0-mm section thickness and no intersection spacing. Images were obtained with an FOV of $14 \times 14$ $\mathrm{cm}$ and a matrix size of $216 \times 192$. Total acquisition time was approximately 40 minutes.

\section{MR Imaging after CAS}

All the patients underwent DWI within 2 days after CAS. DWI was conducted using a spin-echo-type echo-planar imaging sequence with $3 \mathrm{~b}$-values, of 0,500 , and $1000 \mathrm{~s} / \mathrm{mm}^{2}$ along all 3 orthogonal axes and with the following parameters: TR/TE, 3000/80 milliseconds; flip angle, $90^{\circ}$; sensitivity encoding, 3; FOV, $220 \times 220 \mathrm{~mm}$; matrix, $128 \times 128$; section thickness/gap, 5 mm/30\%; scanning time, 35-38 seconds.

\section{CAS Procedure}

The CAS procedures were performed by one interventional neuroradiologist (H.S.K.) with 10 years of experience. Written informed consent for the CAS procedure was obtained from all the patients. Aspirin $(100 \mathrm{mg} / \mathrm{d})$ and clopidogrel $(75 \mathrm{mg} / \mathrm{d})$ were given for a minimum of 3 days before the procedure. All the CAS procedures were performed with the patient under local anesthesia via the percutaneous transfemoral route. Systemic anticoagulation was initiated with a 3000-U bolus of intravenous heparin followed by a 1000-U/h infusion. Routine 3- or 4-vessel cerebral angiography was performed before treatment to evaluate collateral flow. Then, the double coaxial system, which was assembled combining an outer 80-cm-long 8F introducer sheath (Super Arrow-Flex; Teleflex, Limerick, Pennsylvania) and an inner 100-cmlong 8 F guiding catheter (Guider Softip; Boston Scientific, Natick, Massachusetts), was placed in the common carotid artery to enable stent placement. CAS was performed with the Emboshield distal embolic protection system (Abbott Vascular, Abbott Park, Illinois). Predilation was performed with a 4-mm balloon catheter. Deployment of a self-expandable stent (RX Acculink; Abbott Vascular), the size of which was chosen according to the presumed parent size, was performed. Poststenting angioplasty was performed with a 5- to 6-mm-diameter balloon to achieve a residual diameter stenosis of $<20 \%$. After the procedure, all the patients were monitored for 24 hours in the intensive care unit.

\section{Definition and Outcomes}

Carotid plaque imaging was interpreted using plaque analysis software (MRI-PlaqueView; VPDiagnostics, Seattle, Washington) for detection of IPH and maximal wall thickness, and was analyzed by researchers (G.H.C. and S.B.H.) trained in carotid plaque MR imaging and blinded to the study goal (Rev no. 1-1; Rev no. 2-1). ${ }^{14}$ MR-positive IPH was defined as the presence of hyperintense intraplaque of $>200 \%$ of the signal intensity of the adjacent muscle for at least 2 consecutive sections on MPRAGE images. ${ }^{13,15}$ For MPRAGE-positive IPH analysis, signal intensities were measured in a 6 - to $8-\mathrm{mm}^{2}$ circular region of interest over the carotid plaque. Positive DWI for analysis of ipsilateral ischemic lesions was defined as the detection of a hyperintense signal on a DWI trace with an associated signal decrease on the apparent diffusion coefficient map by 2 experienced neuroradiologists through consensus interpretation. Symptomatic carotid artery stenosis was defined as focal neurologic symptoms and DWI-positive imaging that occurred within 1 week of CAS and was attributable to an ipsilateral carotid artery vascular distribution.

Neurologic evaluation was performed before the procedure, immediately afterward, daily after CAS until discharge, and 1 month afterward using the NIHSS and modified Rankin Scale by independent neurologists who were blinded to the CAS procedure.

The primary outcome was the incidence of any stroke, myocardial infarction, or death within 30 days after CAS. A minor stroke was defined as evidence of neurologic deterioration based on an increase of the NIHSS of $<4$ points without the presence of aphasia or hemianopsia, or complete recovery within 1 month. A major stroke was defined as an increase of the NIHSS of $\geq 4$ points, the presence of aphasia or hemianopsia, or any residual deficit beyond 1 month. Hyperperfusion syndrome without intracranial hemorrhage was defined as the occurrence, either alone or in combination, of an ipsilateral throbbing headache with or 
Table 1: Baseline data for patients with MR-positive IPH or without IPH after carotid stenosis

\begin{tabular}{lccc}
\hline & $\begin{array}{c}\text { MR-Positive } \\
\text { IPH }(\boldsymbol{n}=\mathbf{4 3})\end{array}$ & IPH $(\boldsymbol{n}=\mathbf{5 1})$ & $\boldsymbol{P}$ \\
\hline Mean age, y & $74.3 \pm 6.5$ & $72.9 \pm 6.8$ & .324 \\
Men, no. (\%) & $34(79.1)$ & $31(60.8)$ & .074 \\
Right side, no. (\%) & $15(34.9)$ & $23(45.1)$ & .400 \\
Symptomatic event, no. (\%) & $22(51.2)$ & $19(37.3)$ & .213 \\
Mean degree of stenosis, \% & $78.9 \pm 12.4$ & $75.9 \pm 12.3$ & .247 \\
Cerebrovascular history, & & & \\
$\quad$ no. (\%) & & & \\
$\quad$ Previous stroke & $8(18.6)$ & $17(33.3)$ & .159 \\
$\quad$ Previous cardiac disease & $10(23.3)$ & $8(9.8)$ & .433 \\
Cerebrovascular risk factors, & & & \\
$\quad$ no. (\%) & & & \\
Hypertension & $26(60.5)$ & $32(62.7)$ & .835 \\
$\quad$ Diabetes mellitus & $22(51.2)$ & $22(41.5)$ & .535 \\
Hyperlipidemia & $9(20.9)$ & $8(15.7)$ & .595 \\
Current smoking & $16(37.2)$ & $20(39.2)$ & 1.000 \\
\hline
\end{tabular}

without nausea, vomiting, ipsilateral focal seizure, or focal neurologic deficit without radiographic evidence of infarction.

\section{Statistical Analysis}

Continuous values are expressed as means and standard deviations, whereas categoric data are expressed as counts and percentages. Patients were divided by MR positive or negative status and by symptomatic or asymptomatic status. Continuous and categoric variables were compared between groups by using the Mann-Whitney test and the Fisher exact test, respectively. Multivariate analyses for IPH or no IPH, or symptomatic or asymptomatic groups were conducted with logistic regression. Variables with $P<.20$ from univariate analysis were taken as candidate predictors and were entered into a backward-selection algorithm to identify a set of independent predictors. Statistical significance was defined as $P<.05$. All statistical analyses were performed using R 2.14.1 (http://www.r-project.org/).

\section{RESULTS}

\section{Patients}

The mean age of the 94 consecutive patients was 73.6 years (range, 52-87 years), and most patients were men ( $n=68$ [72.3\%]). MR-positive IPH on MPRAGE images was detected in 43 patients $(45.7 \%)$. The baseline data for patients with carotid stenosis with MR-positive IPH or no IPH are shown in Table 1. Symptomatic events with DWI-positive findings were present in 22 patients (51.2\%) in the IPH group and in 19 patients (37.3\%) in the noIPH group. The other baseline data were similar between the IPH and no-IPH groups.

\section{Outcomes between IPH and no-IPH Groups after CAS}

Technical success of the CAS procedure was achieved in all the patients. No procedure-related complications were observed. Data on postprocedural outcomes in the MR-positive IPH and no-IPH groups are shown in Table 2. New postprocedural ischemic lesions on DWI were observed in the hemisphere in 25 patients $(26.6 \%)$. The incidence of ipsilateral new ischemic lesions on DWI was similar between both groups. Hyperperfusion syndromes without intracranial hemorrhage were observed in 11 patients $(11.7 \%)$. Two patients with MR-positive IPH underwent a
Table 2: Postprocedural complications in patients with or without MR-positive IPH

\begin{tabular}{lccc}
\hline & $\begin{array}{c}\text { MR-Positive IPH, } \\
\text { no. (\%) }(\boldsymbol{n}=43)\end{array}$ & $\begin{array}{c}\text { No IPH, } \\
\text { no. (\%) }(\boldsymbol{n}=51)\end{array}$ & $\boldsymbol{P}$ \\
\hline $\begin{array}{l}\text { Hyperperfusion } \\
\text { syndrome }\end{array}$ & $3(7.0)$ & $8(15.7)$ & .218 \\
$\begin{array}{l}\text { lpsilateral ischemic } \\
\text { lesions }\end{array}$ & $9(20.9)$ & $16(31.4)$ & .349 \\
$\begin{array}{l}\text { Minor stroke } \\
\text { Major stroke }\end{array}$ & 0 & 0 & \\
Ml or death & $2(4.7)$ & 0 & .207 \\
\hline
\end{tabular}

Note:-Ml indicates myocardial infarction.

Table 3: Characteristics of symptomatic and asymptomatic groups in patients with carotid stenosis

\begin{tabular}{lccc}
\hline & $\begin{array}{c}\text { Symptomatic } \\
(\boldsymbol{n}=\mathbf{4 1})\end{array}$ & $\begin{array}{c}\text { Asymptomatic } \\
(\boldsymbol{n}=\mathbf{5 3})\end{array}$ & $\boldsymbol{P}$ \\
\hline $\begin{array}{l}\text { Mean age, y } \\
\begin{array}{l}\text { Mean degree of } \\
\text { stenosis, \% }\end{array}\end{array}$ & $74.5 \pm 5.5$ & $72.8 \pm 7.5$ & .214 \\
$\begin{array}{c}\text { MR-positive IPH, } \\
\text { no. (\%) }\end{array}$ & $22(53.7)$ & $19(35.8)$ & .213 \\
$\begin{array}{c}\text { Ipsilateral ischemic } \\
\text { lesions, no. (\%) }\end{array}$ & $17(41.5)$ & $8(15.1)$ & $.005^{\mathrm{a}}$ \\
$\begin{array}{c}\text { Hyperperfusion } \\
\text { syndrome, no. }(\%)\end{array}$ & $5(12.2)$ & $6(11.3)$ & 1.000 \\
$\begin{array}{l}\text { Major stroke, } \\
\text { no. (\%) }\end{array}$ & $1(2.4)$ & $1(1.9)$ & .685 \\
\hline a & & & \\
\hline Fisher exact test. & & &
\end{tabular}

a Fisher exact test.

major stroke within 30 days after CAS. No death or myocardial infarction after CAS was observed. Overall, 30-day stroke, myocardial infarction, and death rates were $2.1 \%$. There was no significant difference in primary outcomes between the MR-positive IPH and no-IPH groups. There were no other independent variables associated with clinical outcomes after CAS between MRpositive IPH and no-IPH groups by univariate and multivariate logistic regression analysis.

\section{Outcome between Symptomatic and Asymptomatic Groups after CAS}

Forty-one patients (43.6\%) were symptomatic. Data on postprocedural outcomes in symptomatic and asymptomatic groups are shown in Table 3. The symptomatic group had a significantly higher incidence of new postprocedural ischemic lesions on DWI than the asymptomatic group ( $41.5 \%$ vs $15.1 \% ; P=.005)$. There was no significant difference in other primary outcomes between the 2 groups. Multivariate logistic regression analysis revealed that postprocedural new ischemic lesions on DWI were found to be independently associated with symptomatic lesions (odds ratio 3.6 [95\% CI, 1.2-2.5]; $P=.010$ ) (Figure).

\section{DISCUSSION}

This study demonstrated that MR-positive IPH on MPRAGE images was not associated with postprocedural outcomes after CAS. Although new postprocedural ischemic lesions on DWI were more frequently found in symptomatic lesions, these were not associated with clinical outcomes after CAS. Therefore, CAS with protection can be performed safely in patients with MR-positive $\mathrm{IPH}$, and the CAS procedure may be carefully performed in symptomatic patients to decrease new postprocedural ischemic lesions on DWI. 

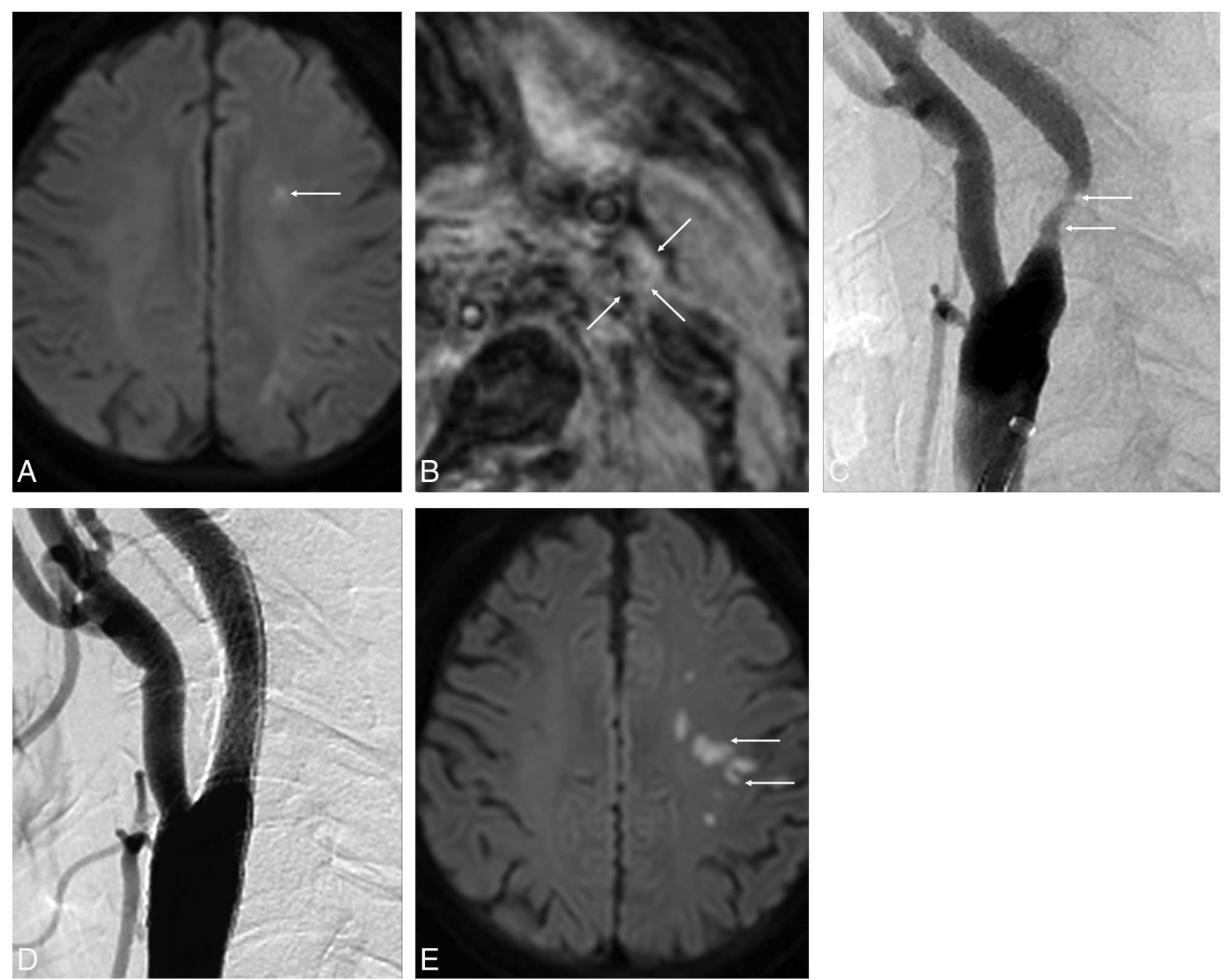

FIGURE. A 77-year-old man with a left acute embolic infarction due to severe stenosis of the left proximal internal carotid artery. A, Axial DWI at admission shows multiple areas of diffusion restriction in the left centrum semiovale (arrow). B, MPRAGE image shows isointensity of the left carotid plaque (arrows). This finding indicates a necrotic core without IPH. C, Lateral projection of left carotid angiography shows moderate stenosis in the proximal cervical portion of the left internal carotid artery (arrows). D, Carotid artery angiogram after protected CAS shows complete recanalization without residual stenosis. E, Axial DWI after CAS shows the new embolic lesions in the left frontal lobe (arrows).

Clinical outcomes of cerebral embolism on TOF images after the CAS procedure in patients with carotid IPH are controversial. A study by Yoshimura et $\mathrm{al}^{11}$ indicated that ischemic events after CAS may be more frequent in plaques that are MR-positive for IPH and that the only independent predictor of postoperative ischemic symptoms was the presence of IPH. Another study from this group performed treatment selection such as CAS or carotid endarterectomy based on MR imaging. ${ }^{14}$ The patients with carotid IPH on TOF images underwent carotid endarterectomy, and the patients without carotid IPH underwent CAS. This study reported that treatment selection based on preoperative MR imaging of the carotid plaque seems useful to reduce postprocedural adverse events after carotid revascularization procedures. This study also indicated that carotid endarterectomy should be selected for MR-positive plaques, whereas CAS should be selected for plaques without IPH to reduce postoperative ischemic symptoms. ${ }^{14}$ However, Yoon et al, ${ }^{12}$ who performed a similar study, reported no significant difference in primary outcomes after CAS between the IPH and no-IPH groups and found no independent variables associated with the primary outcome. However, previous studies from both groups had some limitations. First, these studies used TOF images to evaluate carotid IPH. Sensitivity, specificity, and $\kappa$ values for detection of IPH based on histologic analysis were $80 \%, 97 \%$, and 0.80 for MPRAGE images; 70\%, $92 \%$, and 0.63 for fast spin-echo images; and 56\%, 96\%, and 0.57 for TOF images. ${ }^{13}$ Second, Yoon et $\mathrm{al}^{12}$ did not perform DWI to evaluate new postprocedural ischemic lesions and instead evaluated clinical outcomes after CAS by neurologic examinations. Third, Yoshimura et $\mathrm{al}^{11}$ used variable devices for distal or proximal protection for embolic capture during CAS. Our study was prospectively designed to minimize some bias. All the patients underwent multicontrast MR imaging, including MPRAGE, before CAS. We only used 1 distal embolic protection device and self-expandable stent. All the patients underwent DWI within 2 days after CAS to detect new postoperative ischemic lesions.

Recently, the Carotid Revascularization Endarterectomy Versus Stenting Trial (CREST) reported that CAS and carotid endarterectomy were associated with similar rates of the primary com- 
posite outcomes, periprocedural stroke, myocardial infarction, death, or subsequent ipsilateral stroke among men and women with either symptomatic or asymptomatic carotid stenosis. ${ }^{7}$ However, the incidence of periprocedural stroke was lower in the carotid endarterectomy group, whereas the incidence of periprocedural myocardial infarction was lower in the CAS group. To reduce the risk of stroke after CAS, improvements in training and technique, embolic protection and stent design, and patient selection hold promise. ${ }^{15}$ In our study, 2 patients (4.7\%) with MRpositive IPH experienced a major stroke within 30 days after CAS, but no myocardial infarctions or deaths were observed. Therefore, the overall 30 -day stroke rate was $2.1 \%$. Also, we found no significant difference in primary outcomes after CAS between the MR-positive IPH and no-IPH groups. The similarity of our study and Yoon's study ${ }^{12}$ performed the CAS procedure by 1 interventional neuroradiologist with 10 years of experience and a single product for distal embolic protection.

DWI is a sensitive tool used to identify ischemic lesions after CAS. A previous meta-analysis for detection of ischemic lesions after CAS on DWI revealed a significantly lower incidence $(33 \%)$ in protected patients compared with unprotected patients $(45 \%) .{ }^{16}$ Bijuklic et al ${ }^{17}$ performed pre- and postprocedural DWI for evaluation of new cerebral ischemic lesions in 728 patients undergoing CAS with cerebral embolic protection. New ischemic lesions were found in $32.8 \%$ of the patients. Age, hypertension, lesion length, lesion eccentricity, and aortic arch type III were significantly associated with new ischemic lesions; calcified lesions were negatively associated, which is well in accordance with our findings (25/94 [26.6\%]). In our study, new postprocedural ischemic lesions on DWI were not associated with carotid IPH and were significantly associated with symptomatic lesions, unlike a previous study. ${ }^{11}$ Some studies reported that proximal balloon occlusion reduces embolic events after CAS compared with filter protection ${ }^{18,19}$; however, a high level of technical skill and an experienced interventionalist are very important to prevent new postprocedural ischemic lesions and postprocedural clinical outcomes after CAS. In addition, carotid plaques in patients with symptomatic lesions may have variable vulnerable findings, such as $\mathrm{IPH}$, fibrous cap ruptures, ulcers, thrombus, or inflammation.

Our study had several limitations. First, we lacked a criterion standard histologic reference. Ota et $\mathrm{al}^{13}$ evaluated the diagnostic performance of $3 \mathrm{~T} 1$-weighted $3 \mathrm{~T}$ MR images by using carotid IPH imaging with histologic analysis. The MPRAGE sequence demonstrated a greater ability to detect and quantify IPH than T1-weighted fast spin-echo and TOF sequences. Therefore, we concluded that carotid MPRAGEpositive images strongly indicated the presence of IPH. Second, our study focused on atherosclerotic lesions in the proximal carotid artery. Common causes of acute ischemic stroke are major arterial atheroma, cardioembolic sources, microvascular disease, and cryptogenic factors. Therefore, in our study, symptomatic lesions were defined as focal neurologic symptoms and DWI positive scans that occur within 1 week of CAS because this minimized acute ischemic stroke by other causes. Third, these may have included many causes of vulnerable carotid plaques, such as IPH, fibrous cap rupture, ulcers, throm- bus, large necrotic core, or inflammation. The previous cited studies reported negative clinical outcomes after CAS in patients with carotid IPH. ${ }^{11,12}$ Therefore, our study only focused on carotid IPH for validation. Also, the sample size was relatively small, and long-term follow-up data were not available.

\section{CONCLUSIONS}

The results of this study indicated that IPH in patients with severe carotid stenosis was not a significant risk factor for cerebral embolism after CAS. Symptomatic patients should receive more careful treatment during CAS due to the higher risk of ipsilateral postprocedural ischemic events.

Disclosures: Hyo Sung Kwak—RELATED: Grant: Supported by the Fund of Biomedical Research Institute, Chonbuk National University Hospital.* *Money paid to the institution.

\section{REFERENCES}

1. Timsit SG, Sacco RL, Mohr JP, et al. Early clinical differentiation of cerebral infarction from severe atherosclerotic stenosis and cardioembolism. Stroke 1992;23:486-91 CrossRef Medline

2. Petty GW, Brown RD Jr, Whisnant JP, et al. Ischemic stroke subtypes: a population-based study of incidence and risk factors. Stroke 1999;30:2513-16 CrossRef Medline

3. Palm F, Dos Santos M, Urbanek C, et al. Stroke seasonality associations with subtype, etiology and laboratory results in the Ludwigshafen Stroke Study (LuSSt). Eur J Epidemiol 2013;28:373-81 CrossRef Medline

4. Mas JL, Trinquart L, Leys D, et al. Endarterectomy Versus Angioplasty in Patients with Symptomatic Severe Carotid Stenosis (EVA3S) trial: results up to 4 years from a randomised, multicentre trial. Lancet Neurol 2008;7:885-92 CrossRef Medline

5. International Carotid Stenting Study Investigators, Ederle J, Dobson $\mathrm{J}$, et al. Carotid artery stenting compared with endarterectomy in patients with symptomatic carotid stenosis (International Carotid Stenting Study): an interim analysis of a randomised controlled trial. Lancet 2010;375:985-97 CrossRef Medline

6. Eckstein HH, Ringleb P, Allenberg JR, et al. Results of the Stent-Protected Angioplasty versus Carotid Endarterectomy (SPACE) study to treat symptomatic stenoses at 2 years: a multinational, prospective, randomised trial. Lancet Neurol 2008;7:893-902 CrossRef Medline

7. Brott TG, Hobson RW II, Howard G, et al. Stenting versus endarterectomy for treatment of carotid-artery stenosis. N Engl J Med 2010; 363:11-23 CrossRef Medline

8. Yamada K, Song Y, Hippe DS, et al. Quantitative evaluation of high intensity signal on MIP images of carotid atherosclerotic plaques from routine TOF-MRA reveals elevated volumes of intraplaque hemorrhage and lipid rich necrotic core. J Cardiovasc Magn Reson 2012;14:81 CrossRef Medline

9. Singh N, Moody AR, Gladstone DJ, et al. Moderate carotid artery stenosis: MR imaging-depicted intraplaque hemorrhage predicts risk of cerebrovascular ischemic events in asymptomatic men. $\mathrm{Ra}$ diology 2009;252:502-08 CrossRef Medline

10. Altaf N, Daniels L, Morgan PS, et al. Detection of intraplaque hemorrhage by magnetic resonance imaging in symptomatic patients with mild to moderate carotid stenosis predicts recurrent neurological events. J Vasc Surg 2008;47:337-42 CrossRef Medline

11. Yoshimura S, Yamada K, Kawasaki M, et al. High-intensity signal on time-of-flight magnetic resonance angiography indicates carotid plaques at high risk for cerebral embolism during stenting. Stroke 2011;42:3132-37 CrossRef Medline

12. Yoon W, Kim SK, Park MS, et al. Safety of protected carotid artery stenting in patients with severe carotid artery stenosis and carotid intraplaque hemorrhage. AJNR Am J Neuroradiol 2012;33:1027-31 CrossRef Medline 
13. Ota H, Yarnykh VL, Ferguson MS, et al. Carotid intraplaque hemorrhage imaging at 3.0-T MR imaging: comparison of the diagnostic performance of three T1-weighted sequences. Radiology 2010;254: 551-63 CrossRef Medline

14. Yoshimura S, Yamada K, Kawasaki M, et al. Selection of carotid artery stenting or endarterectomy based on magnetic resonance plaque imaging reduced periprocedural adverse events. J Stroke Cerebrovasc Dis 2013;22:1082-87 CrossRef Medline

15. Chiam PT, Roubin GS, Iyer SS, et al. Carotid artery stenting in elderly patients: importance of case selection. Catheter Cardiovasc Interv 2008;72:318-24 CrossRef Medline

16. Schnaudigel S, Groschel K, Pilgram SM, et al. New brain lesions after carotid stenting versus carotid endarterectomy: a systematic review of the literature. Stroke 2008;39:1911-19 CrossRef Medline
17. Bijuklic K, Wandler A, Varnakov Y, et al. Risk factors for cerebral embolization after carotid artery stenting with embolic protection: a diffusion-weighted magnetic resonance imaging study in 837 consecutive patients. Circ Cardiovasc Interv 2013;6:311-16 CrossRef Medline

18. Stabile E, Sannino A, Schiattarella GG, et al. Cerebral embolic lesions detected with diffusion-weighted magnetic resonance imaging following carotid artery stenting: a meta-analysis of 8 studies comparing filter cerebral protection and proximal balloon occlusion. JACC Cardiovasc Interv 2014;7:1177-83 CrossRef Medline

19. Bijuklic K, Wandler A, Hzizi F, et al. The PROFI study (Prevention of Cerebral Embolization by Proximal Balloon Occlusion Compared to Filter Protection During Carotid Artery Stenting): a prospective randomized trial. J Am Coll Cardiol 2012;59:1383-89 CrossRef Medline 